\title{
Effektivität der Strahlentherapie in der Behandlung von hypophysären Tumoren bei 13 Hunden
}

\author{
Mayer-Stankeova, Simona ; Rohrer Bley, Carla ; Wergin, Melanie ; Sieber-Ruckstuhl, Nadja S ; Wenger, \\ Monique ; Roos, Malgorzata ; Reusch, Claudia E ; Sumova, Andrea ; Kaser-Hotz, Barbara
}

\begin{abstract}
Gegenstand: Zur Evaluation der Wirkung der Strahlentherapie bei großen hypophysären Tumoren und hypophysär bedingtem Hyperadrenokortizismus bei Hunden wurde eine prospektive Studie durchgeführt. Material und Methode: Sieben Hunde wurden nur strahlentherapeutisch behandelt, während sechs andere nach der Strahlentherapie eine medikamentöse Behandlung erhielten. Der Effekt der Strahlentherapie auf die Tumorgröße und die hormonelle Funktion der Hypophyse wurden mithilfe von CT-Kontrollen und ACTH-Stimulationstests beurteilt. Ergebnisse: Eine Verbesserung der neurologischen Symptome trat bei allen Hunden ein. Die Kortisolproduktion musste jedoch mit Medikamenten unterdrückt werden. Eine deutliche Reduktion der Tumorgröße zeigte sich bei den CT-Kontrollen. Die Patienten tolerierten die Bestrahlung der Hypophysentumoren gut. Wesentliche Nebenwirkungen wurden nicht beobachtet. Schlussfolgerung und klinische Relevanz: Strahlentherapie in Kombination mit systemischer Behandlung des Hyperadrenokortizismus zeigte sich effektiv in der Therapie von hypophysären Tumoren bei Hunden. Objective: A prospective study was conducted to evaluate the effect of radiation therapy for treatment of large pituitary tumors and pituitary-dependent hyperadrenocorticism in dogs. Material and methods: Seven dogs received radiation therapy only, whereas six dogs were treated with a combination of irradiation and subsequent medical treatment. The efficacy of radiotherapy on tumor size and function was reviewed by CT control studies and ACTH stimulation tests. Results: Improvement of neurological symptoms occurred in all treated dogs. Reduction of the tumor size was seen on the repeated CT scans. To decrease cortisol production, further medical therapy was needed. Irradiation of the pituitary tumors was well tolerated, no relevant radiation side effects were observed. Conclusion and clinical relevance: Radiation therapy combined with medical treatment of hyperadrenocorticism appears to be efficient in the treatment of pituitary tumors in dogs.
\end{abstract}

DOI: https://doi.org/10.1055/s-0037-1622419

Other titles: Efficacy of radiotherapy in 13 dogs treated for pituitary tumors

Posted at the Zurich Open Repository and Archive, University of Zurich ZORA URL: https://doi.org/10.5167/uzh-19381

Journal Article

Originally published at:

Mayer-Stankeova, Simona; Rohrer Bley, Carla; Wergin, Melanie; Sieber-Ruckstuhl, Nadja S; Wenger, Monique; Roos, Malgorzata; Reusch, Claudia E; Sumova, Andrea; Kaser-Hotz, Barbara (2004). Effektivität der Strahlentherapie in der Behandlung von hypophysären Tumoren bei 13 Hunden. Tierärztliche Praxis. Ausgabe K, Kleintiere, 32(4):232-237.

DOI: https://doi.org/10.1055/s-0037-1622419 


\title{
Effektivität der Strahlentherapie in der Behandlung von hypophysären Tumoren bei 13 Hunden
}

\author{
Simona Mayer-Stankeová1, Carla Rohrer Bley', Melanie Wergin', Nadja Sieber-Ruckstuhl², Monique Wenger², \\ Malgorzata Roos ${ }^{3}$, Claudia Reusch ${ }^{2}$, Andrea Sumová', Barbara Kaser-Hotz' ${ }^{1}$ \\ Aus der ${ }^{1}$ Abteilung für Bildgebende Diagnostik und Radio-Onkologie (Leitung: Prof. Dr. Barbara Kaser-Hotz) und der \\ ${ }^{2}$ Klinik für Kleintiermedizin (Leitung: Prof. Dr. Claudia Reusch) der Vetsuisse-Fakultät der Universität Zürich sowie der \\ ${ }^{3}$ Abteilung für Biostatistik (Leitung: Prof. Dr. Th. Gasser) der Universität Zürich, ISPM
}

Schlüsselwörter:

Hypophysentumoren - Strahlentherapie - Hyperadrenokortizismus - Hund

\section{Zusammenfassung:}

Gegenstand: Zur Evaluation der Wirkung der Strahlentherapie bei großen hypophysären Tumoren und hypophysär bedingtem Hyperadrenokortizismus bei Hunden wurde eine prospektive Studie durchgeführt. Material und Methode: Sieben Hunde wurden nur strahlentherapeutisch behandelt, während sechs andere nach der Strahlentherapie eine medikamentöse Behandlung erhielten. Der Effekt der Strahlentherapie auf die Tumorgröße und die hormonelle Funktion der Hypophyse wurden mithilfe von CT-Kontrollen und ACTH-Stimulationstests beurteilt. Ergebnisse: Eine Verbesserung der neurologischen Symptome trat bei allen Hunden ein. Die Kortisolproduktion musste jedoch mit Medikamenten unterdrückt werden. Eine deutliche Reduktion der Tumorgröße zeigte sich bei den CT-Kontrollen. Die Patienten tolerierten die Bestrahlung der Hypophysentumoren gut. Wesentliche Nebenwirkungen wurden nicht beobachtet. Schlussfolgerung und klinische Relevanz: Strahlentherapie in Kombination mit systemischer Behandlung des Hyperadrenokortizismus zeigte sich effektiv in der Therapie von hypophysären Tumoren bei Hunden.

\section{Einleitung}

Das Cushing-Syndrom oder der Hyperadrenokortizismus stellt bei Hunden eine der am häufigsten diagnostizierten Endokrinopathien dar. Bei 80-90\% der Hunde mit spontanem Hyperadrenokortizismus ist dieser durch eine Überfunktion der Hypophyse bedingt $(6,17)$. Eine übermäßige hypophysäre Sekretion des adrenokortikotropen Hormons (ACTH) wird in der Mehrzahl der Fälle durch Tumoren der Pars distalis oder Pars intermedia der Hypophyse verursacht (15). Die meisten Hypophysentumoren sind hormonell aktiv. Das chronische Übermaß von zirkulierendem Kortisol, bedingt durch die erhöhte hypophysäre Sekretion von ACTH, führt zu typischen Symptomen. Bekannt sind unter anderem Polydipsie, Polyurie, Polyphagie, Hängebauch/Fettsucht, Hecheln, Alopezie, dünnes Haar, Muskelschwäche und Lethargie $(7,16)$.
Key words:

Pituitary tumors - Radiation therapy - Hyperadrenocorticism - Dog

Summary:

Efficacy of radiotherapy in 13 dogs treated for pituitary tumors

Objective: A prospective study was conducted to evaluate the effect of radiation therapy for treatment of large pituitary tumors and pituitary-dependent hyperadrenocorticism in dogs. Material and methods: Seven dogs received radiation therapy only, whereas six dogs were treated with a combination of irradiation and subsequent medical treatment. The efficacy of radiotherapy on tumor size and function was reviewed by CT control studies and ACTH stimulation tests. Results: Improvement of neurological symptoms occurred in all treated dogs. Reduction of the tumor size was seen on the repeated CT scans. To decrease cortisol production, further medical therapy was needed. Irradiation of the pituitary tumors was well tolerated, no relevant radiation side effects were observed. Conclusion and clinical relevance: Radiation therapy combined with medical treatment of hyperadrenocorticism appears to be efficient in the treatment of pituitary tumors in dogs.

Etwa 40-45\% der Hunde mit hypophysär bedingtem Hyperadrenokortizismus haben zur Zeit der Diagnosestellung einen Tumor mit einem Durchmesser von weniger als $3 \mathrm{~mm}$ (2). Neoplasien dieser Größe lassen sich nicht unbedingt mithilfe einer CT oder einer MRT darstellen. Solche Tumoren werden im Allgemeinen als Mikrotumoren bezeichnet, da sie kleiner als $1 \mathrm{~cm}$ im Durchmesser sind (7). Etwa 15-20\% der Hunde mit hypophysär bedingtem Hyperadrenokortizismus entwickeln einen so genannten Makrotumor mit einer Größe von über $1 \mathrm{~cm}$ (5). Diese Tumoren führen aufgrund des Raums, den sie beanspruchen, zur Kompression der umliegenden Strukturen oder sie wachsen invasiv in das umliegende Gewebe ein. Solche Patienten weisen häufig neurologische Symptome von unterschiedlichem Ausmaß auf. Oft wird durch das Tumorwachstum das Bewusstsein beeinflusst, sodass die Patienten Nervosität, Depression, Desorientierung oder Stupor zeigen. Neurologische Defizite wie rotatori- 
Tab. 1 Daten von 13 Hunden mit Hypophysentumoren, die strahlentherapeutisch behandelt wurden. Geschlecht: w = weiblich; wk = weiblich kastriert; $\mathrm{m}=$ männlich; $\mathrm{mk}=$ männlich-kastriert; HAK = Hyperadrenokortizismus; $\mathrm{RT}=$ Radiotherapie; TD = Totaldosis; TV = Tumorvolumen; Fx = Fraktion; ÜZ = Überlebenszeit.

\begin{tabular}{|c|c|c|c|c|c|c|c|c|c|c|c|c|}
\hline Hund & Rasse & $\begin{array}{c}\mathrm{Ge}- \\
\text { schlecht }\end{array}$ & $\begin{array}{c}\text { HAK } \\
\text { vor RT }\end{array}$ & $\begin{array}{l}\text { Neurologische } \\
\text { Symptome vor RT }\end{array}$ & $\begin{array}{l}\text { Dosis pro } \\
\text { Fx (Gy) }\end{array}$ & $\begin{array}{l}\text { Anzahl } \\
\text { Fx (Gy) }\end{array}$ & $\begin{array}{l}\text { TD } \\
\text { (Gy) }\end{array}$ & $\underset{\left(\mathrm{cm}^{3}\right)}{T V}$ & $\begin{array}{l}\text { Trilostan- } \\
\text { Therapie }\end{array}$ & $\begin{array}{c}\text { ÜZ } \\
\text { (Tage) }\end{array}$ & $\begin{array}{l}\text { Grund der } \\
\text { Euthanasie }\end{array}$ & Sektion \\
\hline 1 & \begin{tabular}{|l|} 
Französische \\
Bulldoge
\end{tabular} & w & ja & $\begin{array}{l}\text { Stupor, Kreisbewe- } \\
\text { gung, Drangwan- } \\
\text { dern, Harninkonti- } \\
\text { nenz, Paraparese }\end{array}$ & 3,5 & 11 & 38,5 & 3,88 & nein & 42 & Tumor & nein \\
\hline 2 & Lhasa Apso & $\mathrm{mk}$ & ja & - & 3,5 & 11 & 38,5 & 0,46 & nein & 159 & $\begin{array}{l}\text { Leber- und } \\
\text { Pankreasinsuffizienz }\end{array}$ & $\begin{array}{l}\text { kein Tumor } \\
\text { gefunden }\end{array}$ \\
\hline 3 & \begin{tabular}{|l|} 
Französische \\
Bulldoge
\end{tabular} & $\mathrm{m}$ & ja & - & 3,5 & 12 & 42,0 & 0,61 & ja & 536 & $\begin{array}{l}\text { schlechter Allgemein- } \\
\text { zustand }\end{array}$ & nein \\
\hline 4 & Mittelpudel & wk & ja & - & 3,5 & 12 & 42,0 & 0,33 & ja & 748 & $\begin{array}{l}\text { akuter Durchfall und } \\
\text { Erbrechen }\end{array}$ & nein \\
\hline 5 & \begin{tabular}{|l|} 
Bearded \\
Collie
\end{tabular} & $m$ & ja & $\begin{array}{l}\text { Apathie, } \\
\text { Depression }\end{array}$ & 3,5 & 12 & 42,0 & 0,33 & nein & 1038 & $\begin{array}{l}\text { tot (unklare Ursache), } \\
\text { kein Rezidiv }\end{array}$ & nein \\
\hline 6 & Dalmatiner & $m$ & nein & $\begin{array}{l}\text { Apathie, Depression, } \\
\text { Harninkontinenz }\end{array}$ & 3,5 & 12 & 42,0 & 3,32 & nein & 590 & Durchfall, Erbrechen & nein \\
\hline 7 & Cockerspaniel & $w$ & ja & - & 3,5 & 12 & 42,0 & 0,80 & ja/nein & 937 & lebt & lebt \\
\hline 8 & $\begin{array}{l}\text { West Highland } \\
\text { White Terrier }\end{array}$ & $\mathrm{m}$ & ja & - & 3,5 & 12 & 42,0 & 0,54 & ja & 363 & $\begin{array}{l}\text { akuter Durchfall und } \\
\text { Erbrechen }\end{array}$ & $\begin{array}{l}\text { Adeno- } \\
\text { karzinom }\end{array}$ \\
\hline 9 & \begin{tabular}{|l} 
Labrador \\
Retriever
\end{tabular} & $\mathrm{m}$ & ja & - & 3,5 & 12 & 42,0 & 1,41 & ja & 539 & $\begin{array}{l}\text { Anorexie, schlechter } \\
\text { Allgemeinzustand }\end{array}$ & nein \\
\hline 10 & $\begin{array}{l}\text { Yorkshire } \\
\text { Terrier }\end{array}$ & $\mathrm{m}$ & ja & $\begin{array}{l}\text { Apathie, Para- } \\
\text { parese, Epilepsie }\end{array}$ & 3,5 & 12 & 42,0 & 1,51 & nein & 754 & lebt & lebt \\
\hline 11 & $\begin{array}{l}\text { Deutscher } \\
\text { Boxer }\end{array}$ & $\mathrm{m}$ & ja & Apathie & 3,5 & 12 & 42,0 & 0,68 & ja & 643 & lebt & lebt \\
\hline 12 & Mischling & wk & ja & $\begin{array}{l}\text { Apathie, Para- } \\
\text { parese, Ataxie }\end{array}$ & 3,5 & 12 & 42,0 & 0,85 & nein & 423 & Apathie, Koma & nein \\
\hline 13 & $\begin{array}{l}\text { Labrador } \\
\text { Retriever }\end{array}$ & $\mathrm{m}$ & ja & Apathie & 3,5 & 12 & 42,0 & 1,67 & ja & 315 & $\begin{array}{l}\text { Polydipsie, Polyurie, } \\
\text { schlechter Allgemein- } \\
\text { zustand }\end{array}$ & Karzinom \\
\hline
\end{tabular}

scher Nystagmus, Kreisbewegung, abnormaler Gang (symmetrische Tetraparese, Ataxie) oder das Drängen des Kopfes gegen die Wand werden beobachtet. Im fortgeschrittenen Stadium des Tumorwachstums können auch Verhaltensstörungen (Aggressivität), Defizite der Thermoregulation, neurologische Ausfälle oder Koma auftreten (3, 5, 20, 24, 26). Eine Rassenprädisposition wurde vor allem bei Pudeln, Dackeln und Boxern nachgewiesen. Aber auch Hunde jeder anderen Rasse können daran erkranken $(6,16)$.

Der Erfolg der Strahlentherapie bei großen Hypophysentumoren, die neurologische Symptome und/oder Hyperadrenokortizismus verursachen, wurde beschrieben. Es ergaben sich eine Verbesserung der neurologischen Defizite sowie eine Regression der Tumormasse $(4,8,10,26)$. Die Strahlentherapie soll primär das Tumorwachstum verhindern, da bekannt ist, dass die Bestrahlung die Überfunktion der Hypophyse nicht vollständig beseitigen kann (8). Das Ziel der vorliegenden Studie war, die Wirkung der Strahlentherapie auf die Tumorgröße und auf die Kontrolle der hormonellen Aktivität der Hypophyse bei Hunden mit Hypophysentumoren mit oder ohne Zeichen eines Hyperadrenokortizismus zu beurteilen.

\section{Material und Methoden}

\section{Patienten}

In den Jahren 2000-2002 wurden in der Abteilung für Bildgebende Diagnostik und Radio-Onkologie der Vetsuisse-Fakultät der Universität Zürich 13 Hunde aufgrund einer hypophysären Neoplasie bestrahlt. Die Hunde zeigten entweder neurologische Symptome aufgrund eines großen hypophysären Tumors mit oder ohne Zeichen eines Cushing-Syndroms (Polydipsie, Polyurie, Polyphagie, Alopezie, Hängebauch, Hecheln) oder nur Zeichen eines Hyperadrenokortizismus bedingt durch eine hormonell aktive hypophysäre Masse (Tab. 1).

Bei allen Patienten erfolgten vor der Strahlentherapie eine vollständige klinische Untersuchung, eine Blutuntersuchung sowie eine radiologische Untersuchung der Lungen. Hunde mit Verdacht auf eine große hypophysäre Masse wurden neurologisch abgeklärt und einer computertomographischen Untersuchung (CT) unterzogen. Diejenigen, die mindestens eines der typischen klinischen Symptome eines Cushing-Syndroms zeigten, wurden endokrinologisch und anschließend ebenfalls computertomographisch untersucht. Eine adrenerge Form des Cushing-Syndroms wurde mithilfe einer abdominalen Ultraschalluntersuchung ausgeschlossen.

\section{Computertomographie}

Die CT-Untersuchung (Somatom AR.T, Siemens) fand in der Abteilung für Bildgebende Diagnostik und Radio-Onkologie statt. Es wurden zwei Studien angefertigt, eine Nativstudie und eine Kontrastmittelstudie mit intravenöser Applikation von jodhaltigem Kontrastmittel (Ultravist ${ }^{\circledR}$, Schering AG, Baar, Schweiz; 2,0 ml/kg KM i. v.). Für beide Studien erhielten die Hunde eine Nar- 
kose. Diese wurde mit Midazolam (Dormicum ${ }^{\circledR}$, Roche Pharma AG, Reinach, Schweiz; $0,1 \mathrm{mg} / \mathrm{kg} \mathrm{KM}$ i. v.) und Propofol (Propofol ${ }^{\circledR} 1 \%$ Fresenius, Fresenius AG, Stans, Schweiz; 4,0 mg/kg KM i. v.) eingeleitet und nach Intubation der Tiere mit Isofluran (Forene ${ }^{\circledR}$, Abbott AG, Baar, Schweiz) und Sauerstoff aufrechterhalten. Die Hunde wurden dann sternal mithilfe eines Vakuumkissens in einer Plexiglasbox positioniert (Abb. 1). Zur exakten Lagerung für die CT-Studie und später für die Bestrahlung, musste ein so genannter Beißblock hergestellt werden (22). Für beide Studien wurden $3 \mathrm{~mm}$ dicke, transversale Schnitte mit $1 \mathrm{~mm}$ Vorschub des CT-Tisches angefertigt. Der untersuchte Bereich reichte kaudal vom Dens axis des zweiten Halswirbels bis kranial zur Lamina cribriformis. Beide Studien wurden in einer dorsalen und einer sagittalen Ebene rekonstruiert und die Hypophyse wurde genau evaluiert. Im Anschluss erfolgte mit Hilfe eines computerisierten Planungssystems (Varian CadPlan ${ }^{\circledR}$ ) die exakte Berechnung des Hypophysen- und Gehirnvolumens.

\section{Funktionstests}

Zwölf der 13 Hunde zeigten in unterschiedlicher Intensität Symptome des Cushing-Syndroms. Sie wurden mithilfe des ACTH-Stimulationstests und des niedrig dosierten Dexamethasontests und/oder des Urin-Kortisol-KreatininVerhältnisses (UCC) im Urin getestet (21). Beim ACTH-Stimulationstest kam synthetisches ACTH (Synacthen ${ }^{\circledR}$, Novartis) zum Einsatz. Nach der Entnahme einer Blutprobe für die Bestimmung des Kortisol-Basalwertes erfolgte die Applikation von $0,25 \mathrm{mg}$ ACTH i. m. Eine Stunde später wurde eine zweite Blutprobe zur Bestimmung des Kortisolwertes entnommen. Kortisolwerte im Plasma von $>20 \mu \mathrm{g} / \mathrm{dl}$ eine Stunde nach der ACTH-Gabe wurden als positiv betrachtet (Referenzbereich 5-17 $\mu \mathrm{g} / \mathrm{dl}$ ).

Zur Durchführung des niedrig dosierten Dexamethasontests erhielten die Hunde eine intravenöse Injektion von 0,01 mg/kg KM Dexamethason (Dexamethasone ${ }^{\circledR}$, Virbac). Zuvor wurde eine Blutprobe für den Kortisol-Basalwert entnommen. Für die Analyse der Kortisolkonzentration im Plasma nach der Dexamethasoninjektion wurde vier und acht Stunden später je eine Blutprobe entnommen. Eine Kortisolkonzentration von $\geq 1,4 \mu \mathrm{g} / \mathrm{dl}$ acht Stunden nach Dexamethasongabe galt als positiv (Referenzbereich $<1,4 \mu \mathrm{g} / \mathrm{dl}$ ).

Zur Bestimmung des UCC im Urin wurde eine Morgenurinprobe untersucht. Ein Verhältnis von $>10 \times 10^{-6}$ wurde als positiv beurteilt.

\section{Strahlentherapie}

Die Patienten wurden in der Abteilung für Bildgebende Diagnostik und RadioOnkologie der Vetsuisse-Fakultät der Universität Zürich mithilfe eines 6-MVLinearbeschleunigers (Dynary LA20, ABB, Varian, Baden, Schweiz) mit Photonen bestrahlt. Die Gesamtdosis von 38,5 bis 42,0 Gy wurde in 11 oder 12 Fraktionen von je 3,5 Gy appliziert. Die Strahlentherapie erfolgte über vier Wochen, jeweils am Montag, Mittwoch und Freitag. Sie wurde mit dem Planungsprogramm Varian CadPlan ${ }^{\circledR}$ geplant. Auf der Basis der CT-Studie wurden die Normalstrukturen sowie der hypophysäre Tumor lokalisiert. Die kritischen Organe (Augen, Gehirn), das Tumorvolumen (TV) und das Planungstherapievolumen (PTV) wurden auf jedem Planungsschnitt eingezeichnet. Das PTV beinhaltete das Tumorvolumen und einen Sicherheitssaum von $0,5 \mathrm{~mm}$. Für jede Applikation der Strahlentherapie erhielten die Hunde eine Narkose nach gleichem Narkoseprotokoll wie bei der CT-Studie. Die Position der Hunde bei der Bestrahlung war mit derjenigen der CT-Studie identisch. Der Strahl wurde in insgesamt vier gleich gewichteten Strahlenfeldern auf die Hypophyse gerichtet (Abb. 2).

Vier der 13 Hunde wurden vor der Strahlentherapie wegen ihres Hyperadrenokortizismus mit Medikamenten behandelt: drei Tiere mit Mitotane (Lysodren ${ }^{\circledR}$, Bristol-Myers Squibb AG, Baar), einer mit Trilostan (Modrenal ${ }^{\circledR}$, Wanskerne Ltd, St. Austell, GB). Bei allen Hunden wurde diese Therapie für die Dauer der Strahlentherapie unterbrochen. Sieben Hunde zeigten eine neurologische Symptomatik. Die häufigsten Symptome waren Bewusstseinstörungen (Apathie, $n=6$; Depression, $n=2$; Stupor, $n=1$ ), Verhaltensstörungen (Kreisbewegung, $\mathrm{n}=1$; Drangwandern, $\mathrm{n}=1$; Harninkontinenz, $\mathrm{n}=2$ ) und abnormaler Gang (Paraparese, $n=3$; Ataxie, $n=1$ ). Bei einem Hund wurden epilepti-

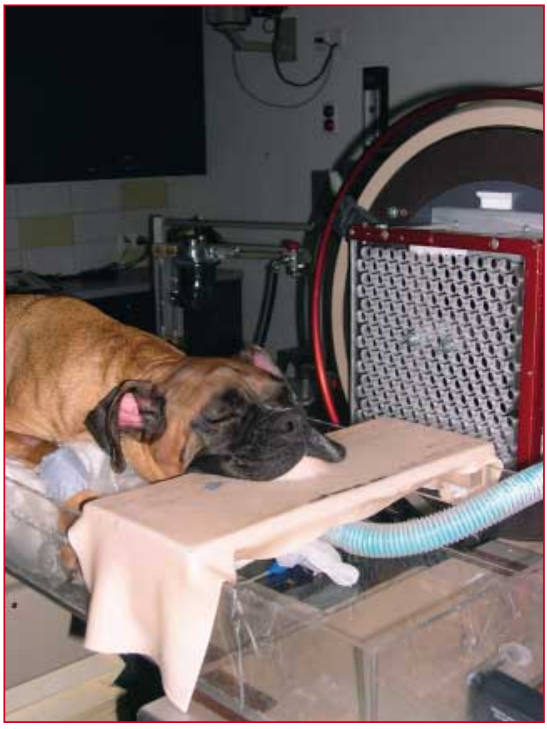

Abb. 1 Narkotisierter Hund positioniert in der Plexiglasbox, bereit für die Strahlentherapie

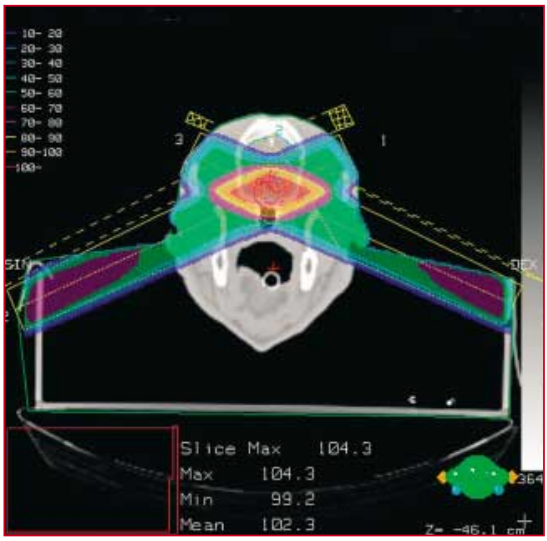

Abb. 2 Dosisverteilung im Bestrahlungsplan von Hund Nr. 11

sche Anfälle beobachtet. Einen, drei, sechs, neun und 12 Monate nach der Strahlentherapie wurden eine klinische Untersuchung, eine Blutuntersuchung und ein ACTH-Stimulationstest durchgeführt. Die CT-Untersuchung zur Bestimmung der Hypophysengröße wurde frühestens sechs Monate nach der Bestrahlung wiederholt.

\section{Statistische Auswertung}

Die statistische Auswertung erfolgte mit dem Statistikprogramm StatView (Version 4.0 statistical software application). Die Überlebenszeit wurde von Anfang der Therapie bis zur Euthanasie berechnet. Eine Signifikanz von folgenden Prädiktoren auf die Überlebenszeit wurde gesucht: Geschlecht, Gewicht, Alter, Tumorvolumen vor der Therapie, Symptome vor der Therapie, neurologische Symptome. Darüber hinaus erfolgte eine Überlebenszeitanalyse unter Verwendung von Kaplan-Meier-Analyse und Longrank-Test sowie Cox Regression. 

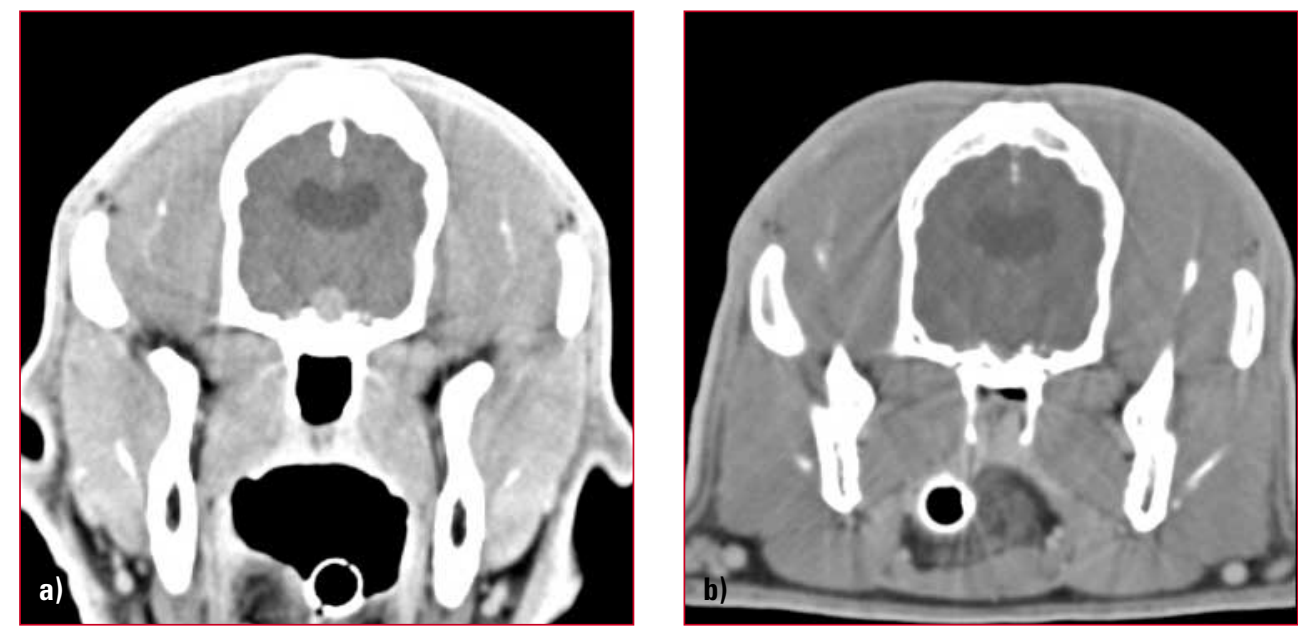

Abb. 3 CT-Aufnahmen des Hundes Nr. 11; a) vor der Strahlentherapie: Transversalschnitt mit einer hyperdensen Masse im Hypophysenbereich. b) sechs Monate nach der Strahlentherapie: Transversalschnitt ohne Masseneffekt im Hypophysenbereich

\section{Ergebnisse}

Das Alter der Hunde lag zwischen 4 und 13 Jahren (Median 9 Jahre), ihr Gewicht variierte von 4 bis $38 \mathrm{~kg}$ (Median $15 \mathrm{~kg}$ ). $69 \%$ (9/13) der Hunde waren männlich, davon einer kastriert, $31 \%$ (4/13) weiblich, davon zwei kastriert. Die Tiere gehörten verschiedenen Rassen an. Französische Bulldogen $(\mathrm{n}=2)$ und Labrador Retriever $(\mathrm{n}=2)$ waren übervertreten (Tab. 1).

Alle Hunde vertrugen die wiederholten Narkosen gut. Während der Strahlentherapie konnten keine wesentlichen und unerwarteten Nebenwirkungen beobachtet werden. In der bestrahlten Region kam es bei keinem Hund zu nässender Dermatitis, Haarausfall oder Verfärbung der Haare. Bei keinem Patienten traten Akut- oder Spätreaktionen des Gehirngewebes auf. Die beschriebenen neurologischen Symptome gingen schon während und kurz nach der Bestrahlung teilweise zurück. Eine weitere Verbesserung konnte bei den Nachkontrollen festgestellt

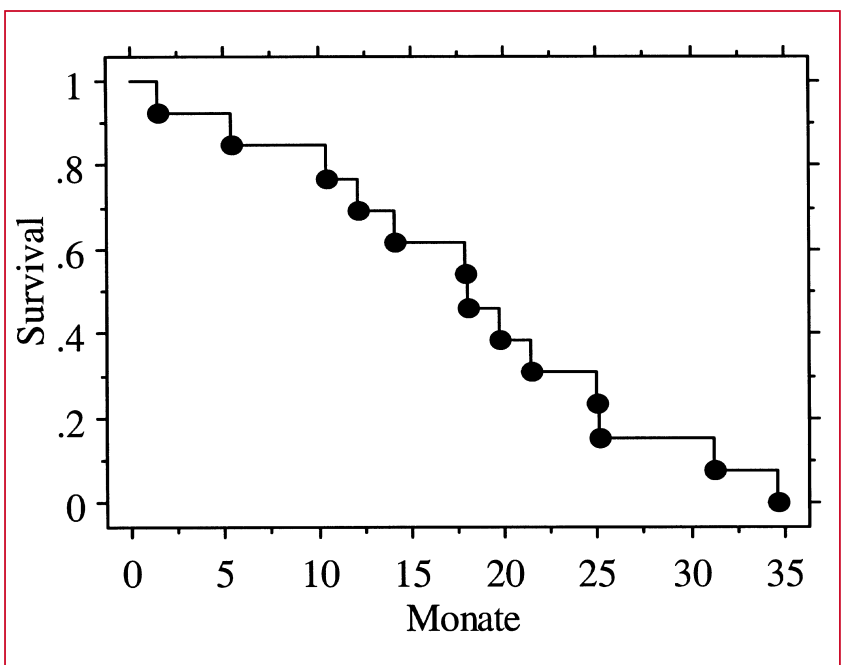

Abb. 4 Überlebenskurve nach Kaplan-Meier für 13 Hunde mit Hypophysentumoren, die strahlentherapeutisch behandelt wurden werden. Bei vier Hunden ließ sich eine komplette Remission der neurologischen Symptomatik nachweisen. Bei zwei weiteren Patienten blieben die Symptome stabil. Einer der Hunde wurde aufgrund der persistierenden Symptome 42 Tage nach der Strahlentherapie auf Wunsch des Besitzers euthanasiert. Bei einem Hund entwickelte sich zwei Wochen nach der Bestrahlung eine Parese des Nervus facialis.

Das Hypophysenvolumen reichte von $0,33-3,88 \mathrm{~cm}^{3}$ mit einem Medianwert von $0,8 \mathrm{~cm}^{3}$. Fünf Hunde hatten einen Tumor größer als $1 \mathrm{~cm}^{3}$ und zeigten eine deutliche neurologische Symptomatik. Bei zwei anderen Hunden mit neurologischen Symptomen betrug das Hypophysenvolumen $0,33 \mathrm{~cm}^{3}$ bzw. $0,85 \mathrm{~cm}^{3}$. Bei fünf Hunden konnte sechs Monate nach der Strahlentherapie eine CT-Kontrolle durchgeführt werden, die eine deutliche Tumorreduktion erkennen ließ (Abb. 3). Diese Abnahme der Tumormasse durch die Bestrahlung war jedoch nicht statistisch signifikant. Das berechnete Tumorvolumen ergab einen Medianwert von $0,14 \mathrm{~cm}^{3}$. Bei einem Hund erfolgte durch den Privattierarzt eine CT-Kontrolle, bei der kein Tumor mehr darstellbar war. Ein Hund wurde aufgrund einer Leber- und Pankreasinzuffizienz 136 Tage nach der Bestrahlung euthanasiert. In der Sektion konnte kein Tumor nachgewiesen werden.

Die Symptome eines Cushing-Syndroms gingen während der Strahlentherapie leicht zurück oder blieben gleich. Bei sieben der 13 Hunde wurde drei bis vier Wochen nach Ende der Strahlentherapie eine Behandlung mit Modrenal ${ }^{\circledR}$ begonnen (23). Unter diesen Tieren befanden sich zwei der vier Hunde, bei denen die medikamentöse Therapie vorübergehend unterbrochen worden war. Einer dieser Hunde wurde nach 12 Monaten weiter mit Lysodren ${ }^{\circledR}$ behandelt. Hinsichtlich der Überlebenszeit bestand kein Unterschied zwischen den Patienten, die mit Modrenal ${ }^{\circledR}$ behandelt wurden, und denjenigen ohne Modrenal ${ }^{\circledR}-$ Gabe.

Die mittlere Überlebenszeit aller Hunde betrug 18 Monate. Nach 18 Monaten lebten noch 50\% der Patienten (Abb. 4). Drei Hunde sind immer noch am Leben (21, 25 und 31 Monate nach der Strahlentherapie) und zeigen keine Anzeichen eines erneuten Tumorwachstums oder eines Rezidivs. Zehn der 13 Hunde star- 
ben oder wurden euthanasiert. Der Grund der Euthanasie ist in Tabelle 1 angegeben. Nur drei von 13 Hunden konnten seziert werden. Bei einem wurde bei der histologischen Untersuchung kein Tumor mehr festgestellt, bei den zwei anderen ließ sich ein Karzinom der Hypophyse nachweisen.

In Bezug auf die Überlebenszeit ergab sich kein Einfluss der Faktoren Rasse, Alter, Gewicht, Geschlecht und sowie Symptome vor der Therapie.

\section{Diskussion}

Zurzeit werden Hunde mit zentralem Hyperadrenokortizismus oder mit einer hypophysären Masse unterschiedlich therapiert. Infrage kommt zum einen eine medikamentöse Behandlung mit Trilostan, Mitotane oder Ketokonazol (7, 13, 23). Die alleinige Gabe von Medikamenten verringert zwar die Symptome des Cushing-Syndroms, kann jedoch die durch eine große, raumokkupierende Masse bedingten neurologischen Symptome nicht verhindern. In der Humanmedizin erfolgt oft eine komplette oder partielle Hypophysektomie, meistens mit einer darauf folgenden Strahlentherapie, die das Risiko einer Rezidivierung minimieren soll (9). In der Veterinärmedizin wird die Chirurgie in Form einer transfrontalen oder transsphenoidalen Hypophysektomie weniger praktiziert, da sie sehr anspruchsvoll und mit einer beträchtlichen Morbidität verbunden ist. Derartige Eingriffe werden hauptsächlich an wenigen spezialisierten Einrichtungen praktiziert $(12,14)$. Die Strahlentherapie wurde mehrmals bei Hunden mit aktiven oder nicht aktiven hypophysären Tumoren angewendet und ihre Bedeutung in der Behandlung dieser Tumoren ist erwiesen (4, 10, 24, 26).

Alle unsere Patienten tolerierten die Strahlentherapie gut und es traten keine bedeutenden Akut- oder Spätreaktionen auf. Aufgrund der physikalischen Eigenschaften des Photonenstrahls und der Dosisverteilung kam es in der bestrahlten Region nicht zu einem Haarausfall. Andere Untersucher stellten nach der Strahlentherapie verschiedene Nebenwirkungen fest wie Haarverlust und Hypopigmentation im bestrahlten Feld, Taubheit, mögliche Neuropathie des Nervus trigeminus oder Otitis externa $(4,8,26)$. In diesen Studien wurde keine computerisierte Therapieplanung verwendet. Dies könnte die schlechtere Dosisverteilung und damit die gravierenden Nebenwirkungen erklären. Ein Hund unserer Studie wies 14 Tage nach der Strahlentherapie eine Parese des Nervus facialis auf. Eine direkte Ursache dafür ließ sich nicht ermitteln. In der Literatur wird eine unilaterale trigeminale Neuropathie drei Jahre nach der Strahlentherapie beschrieben (3). Andere Nebeneffekte der Bestrahlung wie Nekrose, Visusverschlechterung, Gefäßveränderungen und neuropsychologische Auffälligkeiten wurden mehrheitlich bei Humanpatienten beobachtet (1). In der Humanmedizin wurde auch eine Dosisabhängigkeit in Bezug auf die Tumorkontrolle festgestellt $(9,27)$. Heutzutage werden Totaldosen von 45-50,4 Gy empfohlen (1). Um das Risiko einer Gehirnnekrose zu erniedrigen, werden Humanpatienten mit 1,8 Gy pro Fraktion und fünfmal pro Woche bestrahlt $(1,25)$. Das Auftreten einer strahleninduzierten Neopla- sie im bestrahlten Feld wurde beim Menschen beschrieben (11, 18, 19). Das kumulative Risiko, einen sekundären Gehirntumor zu entwickeln, liegt in den ersten 10 Jahren nach der Bestrahlung beim Menschen bei 1,3\% (1). Sekundäre Tumoren entwickeln sich erst nach Jahrzehnten, daher ist dies in Relation zur Lebensdauer des Hundes kaum relevant.

In unserer Studie ließ sich bei allen CT-Nachkontrollen sechs Monate nach Beendigung der Strahlentherapie eine deutliche, mehr als 50\%ige Tumorregression nachweisen (Abb. 3). In einem Fall kam es zu einer vollständigen Rückbildung des Tumors. Die positive Wirkung der Strahlentherapie auf die Hypophysentumoren wurde auch in anderen Studien gezeigt (4, 8, 10, 26). Der am frühesten beobachtete Effekt ist die Rückbildung des peritumoralen Ödems. Eine Regression des Tumors kann noch bis zu einem Jahr nach der Bestrahlung gesehen werden (4). Ein Hund in unserer Studie zeigte so schwere neurologische Symptome, dass er bereits kurz nach der Bestrahlung euthanasiert werden musste. Die Effektivität der Strahlentherapie scheint auch vom Grad der neurologischen Symptome abzuhängen. Die Strahlentherapie war effektiver bei Hunden mit milden oder mäßigen neurologischen Symptomen als bei Tieren mit ausgeprägten Symptomen. Das Tumorvolumen stellte jedoch keinen prognostischen Faktor für die Intensität der neurologischen Symptome dar $(4,26)$. Auch bei Hunden mit großen hypophysären Tumoren kam es zu einer Besserung der neurologischen Symptome und einer deutlichen Reduktion der Tumorgröße (10). Mit einer computertomographischen Untersuchung lassen sich Tumoren früh erfassen. Die CT-Diagnostik sollte eine integrale Rolle in der Aufarbeitung eines Hyperadrenokortizismus spielen. Die Strahlentherapie kann dann zur Prävention der Entwicklung großer Tumoren und neurologischer Symptome bei Hunden mit zentralem Hyperadrenokortizismus eingesetzt werden (8).

Da in der Veterinärmedizin eine Gehirnbiopsie nicht routinemäßig durchgeführt wird, ist eine histologische Diagnose der Hypophysentumoren häufig nicht möglich. Die Diagnose kann meist erst bei der Sektion gestellt werden. Nach Angaben in der Literatur handelt es sich bei hypophysären Tumoren des Hundes hauptsächlich um Adenome (7). In unserer Studie erfolgte bei drei Tieren eine Sektion. Bei zwei Hunden wurde ein Karzinom diagnostiziert, beim dritten ließ sich kein Tumor mehr nachweisen. Die Anzahl der untersuchten Hypophysen war jedoch zu klein, um eine klare Aussage bezüglich der histologischen Befunde machen zu können.

Die Strahlentherapie hatte keinen wesentlichen Einfluss auf die Symptome des Hyperadrenokortizismus. Beim Menschen wird eine Normalisierung der ACTH-Konzentration nach der Bestrahlung beobachtet (4). Dies lässt sich dagegen in der Veterinärmedizin nicht oft feststellen. Deshalb wurden die Tiere mit persistierenden klinischen Symptomen eines Hyperadrenokortizismus medikamentös behandelt. Für viele Besitzer ist vor allem häufiger Harnabsatz des Hundes zu Hause nicht akzeptabel. Aus diesem Grund wird die Medikamentengabe zur Besserung des Hyperadrenokortizismus vier bis acht Wochen nach der Strahlentherapie empfohlen (8). 
Die vorliegende Studie zeigt, dass die Strahlentherapie eine Verringerung der Tumorgröße bewirkt, aber die hormonelle Dysfunktion nicht beheben kann. Die Indikation zur Strahlentherapie ist aber nicht nur als Tumorreduktion zu sehen, sondern auch als präventive Maßnahme, bevor neurologische Symptome infolge der Größe von Hypophysentumoren auftreten.

\section{Literatur}

1. Becker G, Kocher M, Kortmann RD, Paulsen F, et al. Radiation therapy in the multimodal treatment approach of pituitary adenoma. Strahlenther Onkol 2002; 178: 173-86.

2. Bertoy EH, Feldman EC, Nelson RW, Duesberg CA, et al. Magnetic resonance imaging of the brain in dogs with recently diagnosed but untreated pituitary-dependent hyperadrenocorticism. J Am Vet Med Assoc 1995; 206: 651-6.

3. Dow SW, LeCouteur RA. Radiation therapy for canine ACTH-secreting pituitary tumors. In: Current Veterinary Therapy X. Small Animal Practice. Kirk RW, ed. Philadelphia: Saunders 1989; 1031-4.

4. Dow SW, LeCouteur RA, Rosychuk RA, Powers BE, et al. Response of dogs with functional pituitary macroadenomas and macrocarcinomas to radiation. J Small Anim Pract 1990; 31: 287-94.

5. Duesberg CA, Bertoy EH, Feldman EC. Diagnosis und treatment of makrotumors in dogs with pituitary-dependent hyperadrenocorticism. In: Current Veterinary Therapy XII. Bonagura J, ed. Philadelphia: Saunders 1995; 351-5.

6. Feldman EC, Nelson RW. In: Hyperadrenocorticism (Cushing's syndrome). Canine and Feline Endocrinology and Reproduction. Philadelphia: Saunders 1996; 187-91.

7. Feldman EC. Hyperadrenocorticism. In: Textbook of Veterinary Internal Medicine. Ettinger SJ, Feldman EC, eds. Philadelphia: Saunders 2000; 1460-88.

8. Goossens MMC, Feldman EC, Théon AP, Koblik PD. Efficacy of cobalt 60 radiotherapy in dogs with pituitary-dependent hyperadrenocorticism. J Am Vet Med Assoc 1998; 212: 374-6.

9. Grigsby PW, Simpson JR, Emami BN, Fineberg BB, et al. Prognostic factors and results of surgery and postoperative irradiation in the management of pituitary adenomas. Int J Radiat Oncol Biol Phys 1989; 16: 1411-7.

10. Kaser-Hotz B, Stankeova S, Fidel J, Sumova A et al. Strahlentherapeutische Behandlung von hypophysären Makrotumoren bei vier Hunden. Kleintierprax 2001; 46: 197-206.

11. Martin WH, Cail WS, Morris JL, Constable WC. Fibrosarcoma after high energy radiation therapy for pituitary adenoma. Am J Roentgenol 1980; 135: $1087-90$.

12. Meij BP, Voorhout G, Van den Ingh TS, Hazewinkel HA, et al. Results of transsphenoidal hypophysectomy in 52 dogs with pituitary-dependent hyperadrenocorticism. Vet Surg 1998; 2: 246-61.
13. Neiger R, Ramsey I, O'Connor J, Hurley KJ, et al. Trilostane treatment of 78 dogs with pituitary-dependent hyperadrenocorticism. Vet Rec 2002; 150: 799-804.

14. Niebauer GW, Eigenmann JE, Van Winkle TJ. Study of long-term survival after transsphenoidal hypophysectomy in clinically normal dogs. Am J Vet Res 1990; 51: 677-81.

15. Peterson ME, Krieger DT, Drucker WD, Halmi NS. Immunocytochemical study of the hypophysis in 25 dogs with pituitary-dependent hyperadrenocorticism. Acta Endocrinol 1982; 101: 15-24.

16. Peterson ME. Hyperadrenocorticism. Vet Clin North Am Small Anim Pract 1984; 14: 739-49.

17. Peterson ME. Canine hyperadrenocorticism. In: Current Veterinary Therapy IX. Small Animal Practice. Kirk RW, ed. Philadelphia: Saunders 1986; 963-72.

18. Pieterse S, Dinning TA, Blumbergs PC. Postirradiation sarcomatous transformation of a pituitary adenoma: a combined pituitary tumor. Case report. J Neurosurg 1982; 56: 283-6.

19. Powell HC, Marshall LF, Ignelzi RJ. Post-irradiation pituitary sarcoma. Acta Neuropathol 1977; 3: 165-7.

20. Reusch C, Stankeova S, Geissbühler U, Glaus T et al. Zusammenhang zwischen computertomographisch ermittelter Hypophysengröße und dem Auftreten neurologischer Symptome bei Hunden mit Cushing-Syndrom. Kleintierprax 2001; 46: 133-9.

21. Reusch C, Glaus T, Wess G, Tomsa K et al. Klinische, labordiagnostische und spezielle endokrinologische Untersuchungen bei Hunden mit hypophysärem Cushing-Syndrom: ein Vergleich zwischen Hunden mit Makro- und solchen mit Mikrotumoren. Kleintierprax 2001; 46: 261-70.

22. Rohrer Bley C, Blattmann H, Roos M, Sumova A, et al. Assessment of a radiotherapy patient immobilization device using single plane port radiographs and a remote computed tomography scanner. Vet Radiol Ultrasound 2003; 44: 470-5.

23. Ruckstuhl NS, Nett C, Reusch CE. Results of clinical examinations, laboratory tests, and ultrasonography in dogs with pituitary-dependent hyperadrenocorticism. Am J Vet Res 2002; 63: 506-12.

24. Sarfaty D, Carrillo JM, Peterson ME. Neurologic, endocrinologic, and pathologic findings associated with large pituitary tumors in dogs: Eight cases (1976-1984). J Am Vet Med Assoc 1988; 193: 854-6.

25. Sheline GE, Wara WM, Smith V. Therapeutic irradiation and brain injury. Int J Radiat Oncol Biol Phys 1980; 6: 1215-28.

26. Théon AP, Feldman EC. Megavoltage irradiation of pituitary macrotumors in dogs with neurologic singns. J Am Vet Med Assoc 1998; 213: 225-31.

27. Tran LM, Blount L, Horton D, Sadeghi A, et al. Radiation therapy of pituitary tumors: results in 95 cases. Am J Clin Oncol 1991; 14: 25-9.

Prof. Dr. med. vet. Barbara Kaser-Hotz

Abteilung für Bildgebende Diagnostik und Radio-Onkologie

Vetsuisse-Fakultät der Universität Zürich

Winterthurerstrasse 260, CH-8057-Zürich 